\title{
A Precursor on Pandemic Corona Virus: An Infectious Disease
}

\author{
Narmadhadevi $\mathrm{V}^{1}$, Ramya $\mathrm{K}^{2^{*}}$, Kishore Balasubramanian ${ }^{3}$ and Das $\mathrm{J}^{4}$ \\ ${ }^{1} \mathrm{P}$ A College of Engineering and Technology \\ Pollachi, India \\ ${ }^{2} \mathrm{P}$ A College of Engineering and Technology \\ Pollachi, India \\ ${ }^{3}$ Dr. Mahalingam College of Engineering and Technology \\ Pollachi, India \\ ${ }^{4}$ Karunya Institute of Technology and Sciences, Karunya University \\ Coimbatore, India \\ *Corresponding author's email: ramya.vlakshmi [AT] gmail.com
}

\begin{abstract}
This paper is a study on Corona virus and its effects on human beings in particular and how it has become a global pandemic. The reason for the world's pandemic, Corona virus is an RNA virus found extensively in mammals including humans. It is found that the most human corona virus infections cause from mild symptoms to severe respiratory problems and lead to death. In the recent past the world has faced two great epidemics caused by two different beta-corona viruses and they were named as SARS-CoV (Severe Acute Respiratory Syndrome Corona Virus) and (MERS-CoV Middle East Respiratory Syndrome Coronavirus). Despite these viruses had high infectivity, had less mortality rate. As it is known, these types of viruses, in general, cause a mild illness and considerably affect children and young adults. As the whole world is facing a pandemic situation today, carrying out research on finding vaccine to kill the virus is a need of the time. It is obvious that to understand the fighting measurements, in other words readiness of the nation in handling the situation, the local risk assessments are considered to be one of the key factors in comprehending COVID-19. It is pathetic to know that this virus spreads rapidly everywhere and considering this fast outbreak, the global wide readiness, building capacity and mutual collaborations among countries are the most important needs in order to control the outbreak.
\end{abstract}

Keywords- Pandemic, corona virus, infectious diseases, respiratory syndrome, treatment, outbreak control, global readiness.

\section{INTRODUCTION}

It is found that Corona virus is a member of the family of Cronaviridae, they are enveloped and attached to host epithelial cells by the spike (S) envelope proteins $[1,2]$ stated that Fusion will occur either at the plasma membrane or after endocytosis and thereby the genome is translated into a poly-protein processed post translationally and converted into structural proteins which help in forming viral particles and non-structural proteins which abet in the replication of the viral genome.

As found Corona virus causes fatality in animals. The novel virus, which has been pervaded into humans, causes symptoms such as cold, influenza. It has an easy way to spread through droplets when a person coughs or sneezes. When a person touches the droplets (many a time unknowingly) and touches his face, mouth, eyes or nose.

\section{SYMPTOMS}

The symptoms of COVID-19 can range from mild to severe. It is studied that after the exposure to the virus, the symptom takes from 2 to 14 days to develop. The symptoms include fever (The Centre for Disease Control considers a person to have a fever when he or she has a measured temperature of at least $\left.100.4{ }^{\circ} \mathrm{F}\left[38^{\circ} \mathrm{C}\right]\right)$ cough and importantly shortness of breath. [3]

Studies show that people who have weakened immunity may be liable to develop more serious symptoms like pneumonia or bronchitis. [4]. So far, most confirmed cases are in adults and of all other age groups and some children have also been infected. Even though it is stated that children are at lesser risk, the recent studies also find the risk factors for being infected by the virus. The following picture - fig 1, diagrammatically depicts the symptoms of corona virus (NHS) 


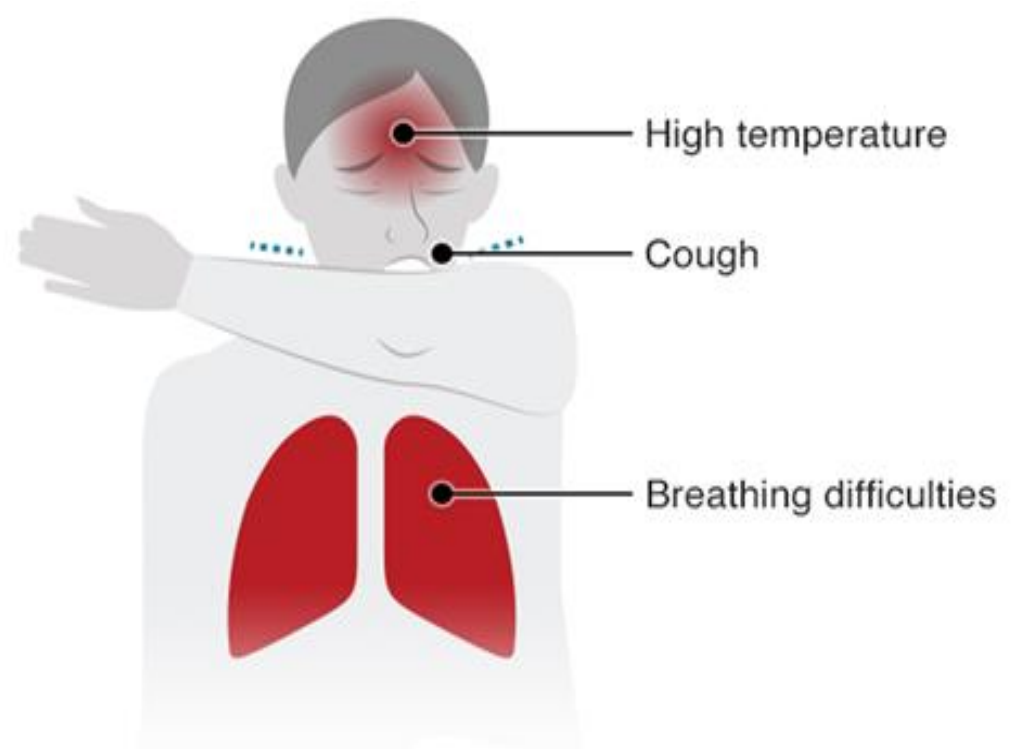

Figure 1: Symptoms of Corona

It has to be unnderstood that the time in/between catching and the showing the symptoms of the corona virus is little higher than the common cold and flu. And the symptom of corona is gradual whereas it is sudden in flu. As the symptos are gradual, they last as well from 2 to 6 weeks from mild to critical. One could differntiate between the common fever as its common symptoms are running nose prevalent in cold and flu and corona wherein breathing difficulty is more common in corona.

\section{COVID - 19 - CRITICAL DISEASE}

The studies reported that around $6 \%$ of infected people become critically ill. As the virus affects the vital organs, they stop functioning and lead to fatality. It is identified that the low-level immunity spiraled out of control that cause damage to the entire body and the organs which results in septic shock and leads to severely drop the blood pressure to low levels and organs stop working properly or fail completely. As it is known, the whole body needs enough oxygen to survive but the corona infected and inflamed lungs - in medical terms - respiratory distress syndrome; will be averted to supply the oxygen to the body. As lungs stop supplying oxygen, the kidneys are thwarted from cleaning the blood and the lining of the intestines are ruined. [5]

It is important to know that the virus causes a greater degree of inflammation and leads to multi-organ failure. If the immune system cannot overpower the virus, there will even be worst complications to the vital organs as the virus spreads to every corner of the body. It is terrible to know that treatment at this stage is highly invasive and ExtraCorporeal Membrane Oxygenation (ECMO) should be included. [6]. But eventually the organs failure occurs and they will be completely failure to the keep the body alive and the damage to the organs leads to the fatal levels.

It is obvious that at this point a question may arise i.e. "Is there a vaccine for corona?" There are many researches are going on to find a vaccine for this virus. So far no vaccine for COVID-19 is reported and there is no antiviral treatment. As stated, prevention is cure, it is necessary to prevent one from the infection and take everyday preventive measures, like maintaining social distance, wearing masks, washing hands with soap frequently, avoiding contacts with sick people and taking foods which increase immunity. People with COVID-19 can seek medical care to help relieve symptoms. [7]

\subsection{Treatment}

It is also important to note that antibiotics are also ineffective in treating COVID-19 because it is a viral infection and not bacterial. If the symptoms are worsening, some of worldwide recommended supportive treatments can be used. [8]. And this supportive treatment may involve based on the condition of the corona victim; they are

- fluids to reduce the risk of dehydration

- medication to reduce fever

- $\quad$ supplemental oxygen in more severe cases and

- providing respirator to those who have a hard time breathing on their own. 


\subsubsection{Some of the preventive remedies for corona virus infection}

The below stated are some of the useful preventive methods which will protect one from getting infected

- Hand wash - It is necessary to wash the hands frequently using soap and warm water or with an alcohol - based hand sanitizer. This will help one keep clean and prevent from any chance of getting infected from the virus. As per the health advisors it is good to wash the hands at least twenty seconds to disinfect.

- Being away from infected people - the most important preventive measure is to avoid close contact with the infected people because it can easily affect people who are in close contact as it is considered to be a communicative disease. As you are avoiding close contacts, it will reduce your chances of getting infected by corona virus. [9]

- Lemon tea - there are studies which recommend to take lemon tea to protect from the virus and helps in treating it. As known, lemon tea is prepared adding lemon juice in black tea or green tea. This removes the virus which causes infection from the passageway and also removes the symptoms of the corona virus, like sore throat.

- Do not smoke - It is advisable not to smoke because it worsens the symptoms of corona virus. It is better to quit smoking and avoid smoking areas.

- Drink plenty of liquids - Hydrating the body is more important as it helps you flush out toxins from the body. Take hot water often and also include herbal tea.

- Stay home and rest - The whole world is in quarantine as they have realized that staying at home is the best remedy for getting infected by the corona virus. It is suggested that people who suffer from corona virus should stay at their homes and to take a good rest. This will help in preventing the virus from spreading to other people and the body will get complete rest as it needs a lot. So staying at home is one of the preventing measures.

- Clean your home - Keeping home clean and tidy is important so as to disinfect the home from viruses and bacteria which will result in decreasing your chances of getting infected. Therefore, keep homes disinfected.

- Cinnamon - As many suggest cinnamon is one of the natural medicines and it can be used in treatment for corona virus. However, it can be taken only by the advice of the doctor as the condition of people varies from one to one.

- Mint tea - it is considered to be a good home remedy for stopping runny nose and helping easy breathing. It can also fight against corona virus.

- Gargle with warm water - it is all time protection from mouth and throat related infections. It clears sore throat. Gargling will help prevent one from getting infected from the virus.

- Garlic - As it has antibacterial and antiviral properties, garlic can be used to fight against corona virus. Taking two cloves of fresh garlic early in the morning will protect one from many viral infections.

- Have your own towel - Practicing hygiene in general is much important phenomenon in one's healthy lifestyle. Hence keeping separate own towel will protect one from getting infected in case anyone is affected at home by the corona virus. When keeping a clean towel that is used just by the person, he can be sure that he is healthy.

- Use a tissue - protecting others from the virus is also important. So one should use tissue to cover his mouth and nose while sneezing and coughing if the person is infected so that he can stop spreading the virus to others.

- Menthol - Using this menthol hot water steaming will relieve one from cold and cough as it has components in many different formulations. It is recommended to add menthol in hot water and inhale which will give relief from cold, cough and nasal congestion.

- Oregano oil - This oregano oil is one of the home remedies available at home. And it is used to relieve from the symptoms. It is important to note that it is not for ingesting but to inhale.

- Thoroughly cook - in general it is really hygiene if the food is completely cooked especially meat and eggs because the virus can also be spread through them also. So cook food thoroughly.

- Avoid unprotected contact with animals - As known, the virus can affect animals too. Therefore, avoid direct and unprotected contact with the farm and wild animals so as to protect you from infection.

- Wear a mask - it is mandatory to all because it is a life-threatening infection as it affects respiratory system. When someone suffers from respiratory infection, then he or she needs to wear a mask, because it can help to protect people around him from illnesses. Use a protective mass to protect you from the infection.

- Lavender oil - this oil also helps cure corona virus when it is inhaled as it is a natural remedy.

- Throw used tissues - It is protective when used tissues are thrown into dustbin or garbage so that spreading the virus can be stopped.

- Keep your hands away - Above all protective measures, it important to note not to touch your mouth, nose and eyes with your hands and fingers, because touching the face may infect you if you suspect that you have, you can touch your face.

- Ginger tea - Some studies show the good effect of ginger tea in treating head ache caused by respiratory infections. So ginger tea as a home remedy can be used to treat corona virus. [10] 
- Salad - the vegetable salads can help detoxify the body and it will help fight corona virus which cause pneumonia. By taking the vegetable salad toxins can be removed. The vegetable used in fighting against are broccoli, cabbage, radishes and beetroot and they detoxify the body

\section{CONCLUSIONS}

The WHO has announced this corona virus spread as world pandemic a worldwide outbreak of disease. People's sustainability is affected when a new virus as the world pandemics emerges to infect people. Since it is a new virus the human pre-existing immunity will not be able to fight against. Corona virus has become a great threat not only to human beings but also to animals. It can easily spread and infect people. It is heartbroken to note that there have many COVID - 19 cases been detected almost in all the countries worldwide and the community spread has also been detected in a growing number of countries. It is recognized as the first pandemic caused by the emergence of a novel corona virus. This world has witnessed four pandemics caused by newly emerged virus influenzas in the past. Since the viruses were life threatening, there were many researches and guidance around the pandemics and the same premises can be applied to the current COVID-19 pandemic.

It is found that in a pandemic intervals framework, the respiratory disease caused by this COVID-19 pandemic follow a certain progression. There are four phases in pandemics such as investigation phase - in the beginning, recognition, initiation, and acceleration phases. It is a point to note that at the end of the acceleration phase the intensity of the disease occurs. And then a deceleration phase wherein the illness is decreased. It is difficult to determine the phases of the pandemic as it varies at any point of time and it is really a bitter truth to know that the different part of the same country can also be in different phases during pandemic. There are many ongoing investigations and research are being carried out to study corona virus and thereby to find out vaccine to destroy the virus. The virus spread is rapid and the information will be updated.

It is obvious that on considering the global burden of respiratory tract infection, there are needs for effective methods of intervention. Vaccination is the only preventive measure or treatment for respiratory virus infection COVID19. There are licensed vaccines available only for influenza. In the absence of mucosal immunity, systemic immune responses can be protective if they are sufficiently robust, such as that induced by inactivated influenza vaccines; however, many vaccines in development for respiratory viruses are live attenuated viruses that are mucosally administered. Though available vaccines show promise, our understanding of the mechanism of protection at mucosal surfaces is incomplete, and there is currently a lack of adequate immune correlates of protection for these vaccines. Besides the immune responses mounted to a natural infection are often not sufficient to prevent reinfection and may also be involved in the pathogenesis of disease. At this point, it is really a challenging task to understand the DNA and RNA of the virus and how it can be broken. To understand its structure has become like herculean task. Even though the virus has become life threatening and has become worldwide pandemic, still there is a hope that vaccine will be found out at the earliest.

\section{CONFLICT OF INTEREST}

Authors declare no potential conflict of interest and funding

\section{REFERENCES}

[1]. Blau D.M., Holmes K.V. Human Coronavirus HCoV-229E Enters Susceptible Cells via the Endocytic Pathway. In: Lavi E., Weiss S.R., Hingley S.T. (eds) The Nidoviruses. Advances in Experimental Medicine and Biology, vol 494. Springer, Boston, MA, 2001.

[2]. Lai MT, et al. Cell growth restoration and high-level protein expression by the promoter of hexose transporter, HXT7, from Saccharomyces cerevisiae. Biotechnol Lett vol 29, no 8, pp: 1287-92, 2007

[3]. Xu X, Chen P, Wang J, et al., Evolution of the novel coronavirus from the ongoing Wuhan outbreak and modeling of its spike protein for risk of human transmission. Life Sciences ; vol 63, no 3, pp:457-460 , 2020

[4]. Guan W, Ni Z, Liang W, et al. Clinical characteristics of coronavirus disease in China. N Engl J Med February 28, 2020; DOI:10.1056/MEJMoa2002032.

[5]. Coronavirus. https://www.webmd.com/lung/coronavirus\#1. Published January 22, 2020.

[6]. https://www.aljazeera.com/news/2020/01/coronavirus-symptoms-vaccines-risks-200122194509687.html . Published January 30, 2020

[7]. https://www.marham.pk/all-diseases/coronavirus

[8]. Jacobs, S.E., Lamson, D.M., et al. Human rhinoviruses. Clin. Microbiol. Rev. vol 26, pp 135-162, 2013

[9]. Bella, J., Rossmann, M.G., ICAM-1 receptors and cold viruses. Pharm. Acta Helv. 74, 291-297, 2000.

[10]. Han, T., Marasco, W.A., Structural basis of influenza virus neutralization. Ann. N.Y. Acad. Sci. 1217, pp 178190, 2011 